\title{
DIRECT RENIN INHIBITORS IN ARTERIAL HYPERTENSION TREATMENT
}

\author{
Viktor Meretskyi \\ Prof., DSc, MD, Ternopil State Medical University by I.Ya. Horbachevskyi, \\ e-mail: meretskyy@tdmu.edu.ua, orcid.org/0000-0002-6358-6884, Ukraine \\ Iryna Meretska \\ Associate Professor, PhD, MD, Ternopil State Medical University by I.Ya. Horbachevskyi, \\ e-mail: meretskaiv@tdmu.edu.ua, orcid.org/0000-0002-2953-903X, Ukraine

\section{Olena Kulyanda} \\ Assistant, PhD, MD, Ternopil State Medical University by I.Ya. Horbachevskyi, \\ e-mail: kulyanda_olol@tdmu.edu.ua, orcid.org/0000-0001-6197-9046, Ukraine

\section{Georges Kamtoh} \\ $\mathrm{PhD}, \mathrm{MD}$, Polonia University in Czestochowa, \\ e-mail: georges2209@yahoo.fr, orcid.org/0000-0002-7897-5831, Poland
}

\begin{abstract}
High blood pressurewas the leading cause of death and disability-adjusted life years worldwide. Despite progress in treatment of hypertension, a number of people with uncontrolled or resistant hypertension increases. Hypertensive disorders are strongly linked with an overactive renin-angiotensin aldosterone system. The renin-angiotensin-aldosterone system has been a highly successful pharmacologic target, as the system is strongly implicated in the development of hypertension-related target organ damage.renin-angiotensin aldosterone systemrenin-angiotensin aldosterone system Aliskiren, an octanamide, is the firstof a new class of completely non-peptide, low-molecular-weight, orally active transitionstate renin inhibitors and only approved for the treatment of hypertension.
\end{abstract}

Keywords: hypertension, renin-angiotensin aldosterone system, renin inhibition.

DOI: http://dx.doi.org/10.23856/2515

\section{Introduction}

One of the key risk factors for cardiovascular disease (CVD) is hypertension (HT) - or raised blood pressure (BP), defined as $\mathrm{SBP}>140 \mathrm{~mm} \mathrm{Hg}$ or DBP > $90 \mathrm{~mm} \mathrm{Hg}(>130 \mathrm{~mm}$ $\mathrm{Hg}$ or $>80 \mathrm{~mm} \mathrm{Hg}$ byHigh Blood Pressure Clinical Practice Guideline (ACC/AHA, 2017). High BP was the leading cause of death and disability-adjusted life years worldwide. Hypertension is a global public health issue, already affects one billion people worldwide, leading to heart attacks, strokes, kidney failure and premature mortality and disability.In the United States, hypertension (ACC/AHA, 2017) accounted for more CVD deaths than any other modifiable CVD risk factor and was second only to cigarette smoking as a preventable cause of death for any reason.Researchers have estimated that raised blood pressure currently kills nine million people every year (WHO, 2013).

The prevalence of hypertension appears to be around 30-45\% in adults over 25 years old of the general population, with a steep increase with ageing. There also appear to be noticeable differences in the average BP levels across countries.Western European countries exhibit a downward trend, in contrast to eastern European countries, which show a clear-cut increase in death rates from stroke (one of the most important hypertension outcomes) (ESH/ESC Guidelines, 2013). The increasing prevalence of hypertension is attributed to population growth, ageing and behavioural risk factors, such as unhealthy diet, harmful use of 
alcohol, lack of physical activity, excess weight and exposure to persistent stress. In addition, there are several metabolic factorsincluding diabetes and high cholesterol, that increase the risk ofcomplications of hypertension (WHO, 2013).

Evidence favouring the administration of BP-lowering drugs to reduce the risk of major clinical cardiovascular (CV) outcomes (fatal and non-fatal stroke, myocardial infarction, heart failure and other $\mathrm{CV}$ deaths) in hypertensive individuals results from a number of randomized controlled trials (RCTs) - mostly placebo-controlled (ESH/ESC Guidelines, 2013).

According to current ESH (European Society of Hypertension)/ESC (European Society of Cardiology) guidelines, physician have at their disposal five main groups of antihypertensives (as also referred to as basic antihypertensives) for use in either monotherapy or in combination: diuretics, beta-blockers $(\mathrm{BBs})$, calcium ion channel blockers (CCBs), angiotensin-converting enzyme inhibitors (ACEIs) and angiotensin II type I ( $\left.\mathrm{AT}_{1}\right)$ receptor blockers (ARBs) - sartans (Vaclavik, Sliva, 2014).

Despite progress in treatment of HT, a number of people with uncontrolled or resistant HT increases. There is a problem of inefficiency of therapy or lack of patients' adherence to treatment. Possibly, poor compliance on the part of the patients is responsible. Another possibility is that awareness of prevention strategies and their implementation by primary physicians may not always be optimal. Finally, some lay the blame at the feet of the health care system or third-party payers. Undoubtedly, all of these factors play a role. However, an additional explanation might be that we need better drugs for patients with essential hypertension. Therefore, search for new approaches to treatment of HT continues (Popov, Bulanov, Ivanov, 2012). In any given patient, several concomitant pharmacologic targets must be addressed. Indeed, patients who require four or more classes of drugs to achieve the current treatment guidelines are no rarity (Müller, Luft, 2006).

\section{Renin-angiotensin aldosterone system}

Hypertensive disorders are strongly linked with an overactive renin-angiotensin aldosterone system (RAAS), and RAAS inhibitors (Ferrari, 2013). The renin-angiotensin aldosterone system plays an integral role in the homeostatic control of arterial pressure, tissue perfusion, and extracellular volume (Atlas, 2007; Katsi et al., 2015). The renin produced by the juxtaglomerular cells in the kidney cleaves angiotensinogen, which is produced by the liver, to release the inactive polypeptide angiotensin I (Ang I). Ang I is converted by angiotensin-converting enzyme into the polypeptideangiotensin II (Ang II). Ang II is the effector enzyme of the cascade and most biological actions of Ang II are mediated primarily through type $1\left(\mathrm{AT}_{1}\right)$, and type II $\left(\mathrm{AT}_{2}\right)$ receptors. In human activation of $\mathrm{AT}_{1}$ receptors causes vasoconstriction via activation of phospholipase and inhibition of adenylate cyclase. Increased blood pressure, promotes adrenal aldosterone secretion, renal sodium reabsorbtion and release of catecholamine from the adrenal medulla and prejunctional nerve endings (Macit, Mercanoglu, 2015). The induced effects include vasoconstriction, sodium and water retention, decreased renal perfusion and cardiac and vascular hypertrophy (Morishita, Kusano, 2013).

There are well-established drugs that interfere with RAAS at several sites, including (1) ACEIs, (2) ARBs, (3) direct renin inhibitors (DRIs), (4) mineralocorticoid receptor antagonists (MRAs), and even (5) beta blockers, the last of which may be considered partial inhibitors (Ghazi, Drawz, 2017; Ferrari, 2013). ACEIs and ARBs have been the cornerstone 
of RAAS inhibition for years and are key therapeutic options in patients with hypertension, reducing cardiovascular morbidity and mortality and improving renal outcomes (Ghazi, Drawz, 2017). However, inhibition of RAAS with ACEIs or ARBs has proven effective for controlling hypertension most important handicap of these agents is incomplete blockage of RAAS. A fundamental reason is reduced feedback inhibition of renin release, triggering a reactive rise in plasma renin activity. With an ACEIs, the reactive rise in plasms renin activity causes compensatory increase in Ang I, which partially restores Ang II production by both ACE-dependent and independent pathways (ACE-escape phenomenon) (Macit, Mercanoglu, 2015).

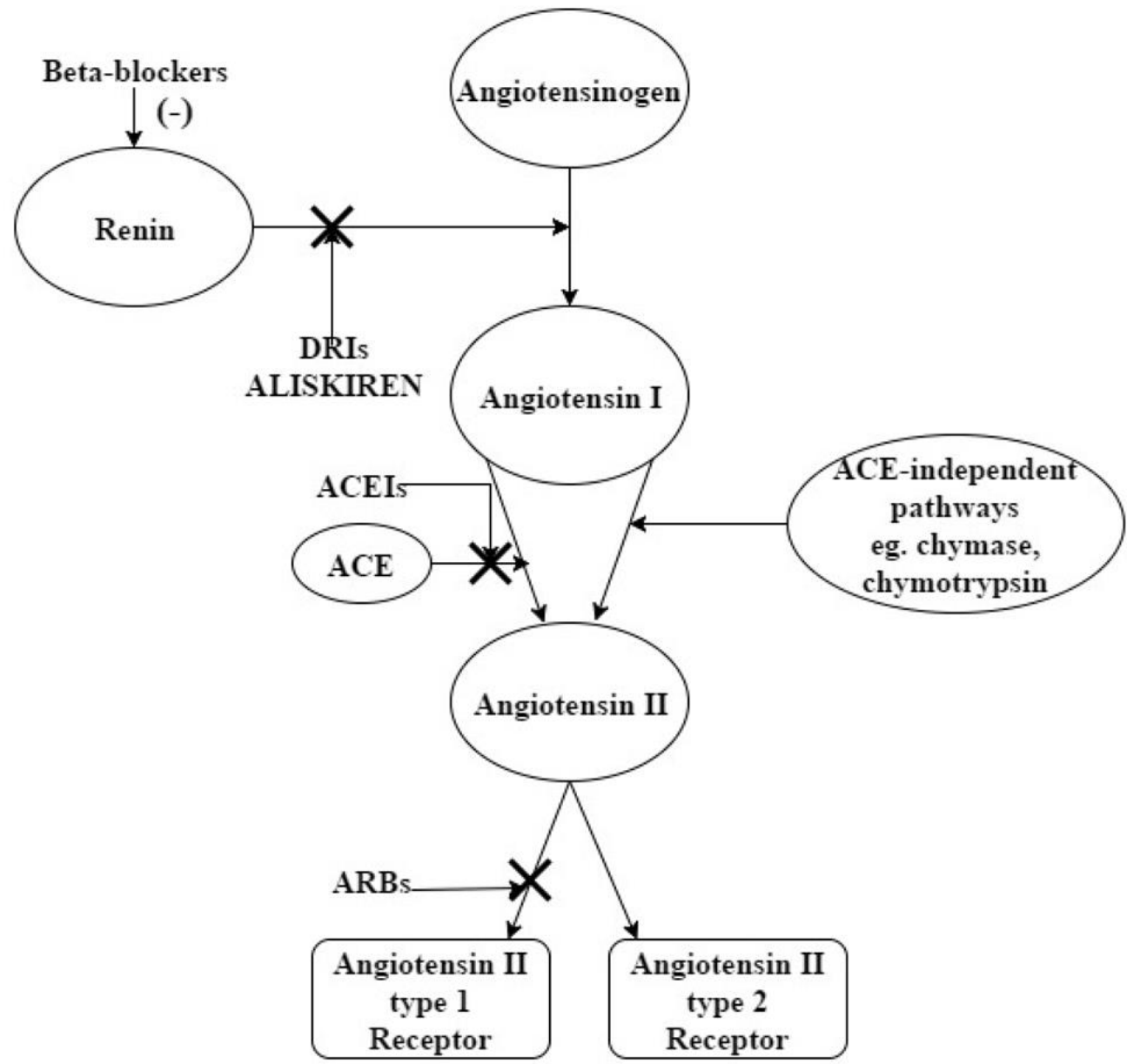

Fig. 1. Schematic representation of the renin-angiotensin-aldosterone system and its pharmacological blockade

(Pantzaris, Karanikolas, Tsiotsios, Velissaris, 2017)

Renin secretion, a rate-limiting step, is the first step of the RAAS cascade, and thus represents the most logical target for inhibition of the renin system. Whereas angiotensinogen is present at plasma concentrations of approximately 500 to $600 \mathrm{pmol}$, Ang I is present in the 
50- to 100-fmol range and Ang II at approximately half that. From a pharmacologist's perspective, the renin step would be the one earning the target focus, as it is the step with the largest step down in concentration (Müller, Luft, 2006). Renin has a unique specificity for its substrate angiotensin. Inhibition of renin provides an attractive option to inhibit the RAAS from its origin (Ghazi, Drawz, 2017). By complete blockage of RAAS at its origin, decrease in both Ang I and Ang II levels can be achieved by DRIs. Although blocking feedback inhibition causes reactive rise in renin secretion, plasma renin activity, the enzymatic activity of renin is markedly reduced by the DRI (DRI binds directly to the catalytic site of renin). Therefore, through this more complete RAAS inhibition, DRIs can offer greater protection from hypertensive complications (Müller, Luft, 2006; Musini et al., 2017).

The development of DRI started more than 30 years ago (Ghazi, Drawz, 2017). The first synthetic renin inhibitor was pepstatin, which was followed by first-generation agents that were active but required parenteral administration. In 1980s, first orally active direct renin inhibitor compounds were developed, such as enalkiren, remikiren, and zankiren. Because of their poor bioavability (less than $2 \%$ ), short half-lives and weak antihypertensive activity they had limited clinical use (Macit, Mercanoglu, 2015).

\section{Blood Pressure Lowering with Aliskiren}

Aliskiren,an octanamide, is the first (approved in 2007) of a new class of completely non-peptide, low-molecular-weight $(609.8 \mathrm{Da})$, orally active transition-state renin inhibitors and only approvedfor the treatment of hypertension (Pantzaris, Karanikolas, Tsiotsios, Velissaris, 2017; Nissenson, Fine, 2017), and a significant BP reduction has been demonstrated in patients with essential HT with no rebound effects on BP after the withdrawal of the treatment (Ghazi, Drawz, 2017; Wal, Rai, Dixit, 2011).

By binding to renin with high affinity, aliskiren blocks the conversion of angiotensinogen to angiotensin I, which subsequently results in a reduction in angiotensin II concentrations. Unlike the angiotensin-converting enzyme inhibitors and the angiotensin II receptor blockers, which reactively stimulate an increase in plasma renin activity, aliskiren suppresses the effects of renin and leads to a reduction in plasma renin activity (Sanoski, 2009).

Aliskiren has good water solubility (this property is important prerequisite for improved oral bioavailability) and low level of lipophilicity and is resistant to biodegradation by peptidases found in the intestine, circulation and/or the liver (Stanton, Jensen, Nussberger, O'Brien, 2003). Although aliskiren exhibits oral bioavailability compared with other previously synthesized renin inhibitors, it is still poorly absorbed (oral bioavailability, $2.5 \%$ ) (Wal, Rai, Dixit, 2011). Aliskiren is 50\% protein bound, and the apparent volume of distribution is $135 \mathrm{~L}$ (Waldmeieret and al., 2007). The plasma concentrations of aliskiren peak between 2 and $4 \mathrm{~h}$ following its administration and steady state is reached after 5-8 days of once-daily administration (Staessen, Li, Richart, 2006; Pool, 2007). Aliskiren demonstrates dose-linear pharmacokinetics over the dose range 75-600 mg in healthy volunteers (Sica, 2009). It has a long half-life (approximately $40 \mathrm{~h}$ ) and provides a 24-hour antihypertensive effect with once-daily dosingwith less potential for loss of efficacy between doses than shorter acting agents (Saseen, 2013). Following oral administration, aliskiren undergoes minimal metabolism (based on in vitro studies, aliskiren is metabolized by CYP 3A4) and is mainly eliminated as unchanged (mostly unabsorbed) compound in the feces. The pharmacokinetics of aliskiren are not effected by moderate to 
severe chronic kidney disease or hepatic impairment. Aliskiren has a low potential for drug interactions (including warfarin, digoxin, statins and other antihypertensive agents, celecoxib, cimetidine) relating to its modest protein binding, limited metabolism as well as its lack of effect on a wide range of $\mathrm{CYP}_{450}$ isozymes (Sica, 2009, Macit, Mercanoglu, 2015). Coadministration of aliskiren with furosemide reduced the AUC of furosemide by $28 \%$ and $\mathrm{C}_{\max }$ by $49 \%$, but clinical significance of this remains uncertain.

Effects of other drugs on aliskiren

Irbesartan: Coadministration of irbesartan reduced aliskiren $\mathrm{C}_{\max }$ up to $50 \%$ after multiple dosing. P-glycoprotein Effects: Pgp (MDR1/Mdr1a/1b) was found to be the major efflux system involved in absorption and disposition of aliskiren in preclinical studies. The potential for drug interactions at the Pgp site will likely depend on the degree of inhibition of this transporter.

Atorvastatin: Coadministration of atorvastatin resulted in about a $50 \%$ increase in aliskiren $\mathrm{C}_{\max }$ and $\mathrm{AUC}$ after multiple dosing.

Ketoconazole: Coadministration of $200 \mathrm{mg}$ twice-daily ketoconazole with aliskiren resulted in an approximate $80 \%$ increase in plasma levels of aliskiren. A 400-mg oncedaily dose was not studied but would be expected to increase aliskiren blood levels further.

Itraconazole: Coadministration of $100 \mathrm{mg}$ itraconazole with $150 \mathrm{mg}$ aliskiren resulted in approximately 5.8-fold increase in $\mathrm{C}_{\max }$ and 6.5-fold increase in AUC of aliskiren. Concomitant use of aliskiren with itraconazole is not recommended.

Cyclosporine: Coadministration of 200 and $600 \mathrm{mg}$ cyclosporine with $75 \mathrm{mg}$ aliskiren resulted in an approximately 2.5 -fold increase in $\mathrm{C}_{\max }$ and fivefold increase in AUC of aliskiren. Concomitant use of aliskiren with cyclosporine is not recommended.

Verapamil: Coadministration of $240 \mathrm{mg}$ of verapamil with $300 \mathrm{mg}$ aliskiren resulted in an approximately twofold increase in $\mathrm{C}_{\max }$ and AUC of aliskiren. However, no dosage adjustment is necessary (Wal, Rai, Dixit, 2011).

Renin inhibition with aliskiren reaches above $99 \%$ in the first hours following administration and remains above 95\% $24 \mathrm{~h}$ later (Pantzaris, Karanikolas, Tsiotsios, Velissaris, 2017). Aliskiren was generally well tolerated, and produced sustained BP reductions in patients with hypertension during 6 months, greater than those with ramiprilbased therapy.

The incidence of adverse events with aliskiren and the number of study discontinuations as a result of adverse events during aliskiren treatment have been relatively low and were similar to results obtained in patients treated with placebo (Allikmets, 2007). Most commonly reported adverse effects are headache, diarrhea, dizziness, fatigue, and back pain. Furthermore because of having no interaction with metabolism of substance $\mathrm{P}$ and bradykinin, cough and angioedema like adverse effects caused by the use of ACEIs, do not occur with aliskiren treatment (Macit, Mercanoglu, 2015). Aliskiren had no clinically important effects on total cholesterol, high-density lipoproteins, fasting triglycerides, or fasting glucose. Laboratory abnormalities that may occur in some patients include a minor increase in blood urea nitrogen and serum creatinine, small reductions in hemoglobin and hematocrit, an increase in serum potassium greater than $5.5 \mathrm{mEq} / \mathrm{L}$, elevated uric acid levels, and renal stones (Wal, Rai, Dixit, 2011).

Aliskiren is available in 150- and 300-mg tablets. The usual recommended starting dose of aliskiren is $150 \mathrm{mg}$ QD. Doses $>300 \mathrm{mg}$ did not provide an increased BP response but did increase the rate of diarrhea (Pantzaris, Karanikolas, Tsiotsios, Velissaris, 2017). The antihypertensive effect of a given dose of aliskiren is attained after 2 weeks of therapy. No 
dosage adjustment is required when used in elderly patients (i.e., those aged $>65$ years) or those with mild to severe renal impairment (creatinine clearance, $<80 \mathrm{~mL} / \mathrm{min}$ ) or hepatic impairment (Child-Pugh Clinical Assessment score, 5-15) (Wal, Rai, Dixit, 2011).

Rebound hypertension has not emerged as a problem with aliskiren. Theoretically it is conceivable that long-term renin-inhibition therapy could induce pharmacologic tolerance with renin hypersecretion as well as the phenomenon of rebound hypertension after abrupt cessation of chronic therapy. However, clinical experience with aliskiren does not confirm this (Allikmets, 2007).

Several randomized control trials conducted showed significant dose-related BP lowering effects with aliskiren monotherapy, similar to those observed with losartan (LOS), valsartan (VAL), irbesartan (IRB), and lisinopril (LIS), and a placebo-like tolerability profile.Severalmeta-analyses published concluded that aliskiren is equally effective with ARBs with a similar adverse effects profile and is as effective as ARBs although it had higher control rates.Aliskiren was also proven superior to ACEIs in diastolic BP reductions, similar to hydrochlorothiazide (HCTZ), and inferior to $\mathrm{CCBs}$ in $\mathrm{BP}$ reduction and control rates (Pantzaris, Karanikolas, Tsiotsios, Velissaris, 2017).

The combination therapy of aliskiren and CCBs, diuretics, ACEIs and ARBs may have synergistic anti-hypertensive effects (Morishita, Kusano, 2013). Aliskiren also neutralizes the reactive PRA increase caused by HCTZ.Studies of aliskiren/amlodipine combinations showed that doses of 300-150/10 $\mathrm{mg}$ are more effective than amlodipine $10 \mathrm{mg}$ monotherapy and have a significantly lower incidence of peripheral edema.Liu et al., in 2014showed that combination therapies aliskiren with amlodipine or HCTZ were more efficient than the respective monotherapies and that aliskiren/amlodipine produced significantly greater SBP/DBP reductions, and higher response and control rates. Triple combinations with aliskiren/amlodipine /HTCZ 300/10/25 mg have also shown similar tolerability and higher efficacy with significantly larger $\mathrm{msSBP} / \mathrm{msDBP}$ reductions and higher control rates as compared to the components' dual combinations in patients with moderate-to-severe hypertension (Pantzaris, Karanikolas, Tsiotsios, Velissaris, 2017).

According to current data, the combination of aliskiren and ACEIs or ARBs is not recommended for hypertensive patients with diabetes, CVD or at least renal disease, especially when considering the risk of developing new adverse events such as hyperkalemia, renal dysfunction and hypotension (Sen, Ufuktepe, Ozunal, Uresin, 2014; Macit, Mercanoglu, 2015).

Hyperkalemia is the primary danger associated with RAAS blocking medications. The RAAS blockade leads to a decrease in aldosterone levels. Since aldosterone has a central role in the excretion of potassium, the RAAS blockers can cause retention of potassium. However, since the benefits of RAAS inhibitors outweigh the risks of hyperkalaemia, there remains the need to overcome these challenges rather than withdraw treatment (ESC, 2016).

The addition of aliskiren to standard optimal therapy (ACEIs or ARBs and betablocker) in post-MI patientsdid not produce any change in left ventricular end-systolic volume compared to placebo. No benefit was shown by the use of aliskiren in prehypertensive individuals with coronary atherosclerosis (Pantzaris, Karanikolas, Tsiotsios, Velissaris, 2017) or improvement in cardiovascular outcomes in patients hospitalized with heart failure (Ghazi, Drawz, 2017) was seen with aliskiren compared with placebo. 


\section{Conclusions and suggestions}

Hypertension is the most common condition seen in primary care and leads to myocardial infarction, stroke, renal failure, and death if not detected early and treated appropriately. Evidence from randomized controlled trials has shown benefit of antihypertensive drug treatment in reducing important health outcomes in persons with high $\mathrm{BP}$ using diuretics, BBs, CCBs, ACEIs and ARBs. The renin-angiotensin aldosterone system plays an integral role in the homeostatic control of arterial pressure. However, adequate RAAS blockade cannot be achieved with ACE inhibitors or ARBs because of incomplete blockage of RAAS.The primary rate-limiting step in the RAAS now can be pharmacologically inhibited directly. Aliskiren, an octanamide, is the orally active transitionstate renin inhibitors and only approved for the treatment of hypertension. Aliskiren is effective in reducing blood pressure and is well tolerated, with a side-effect profile similar to placebo or ACEIsand ARBs. The combination therapy of aliskiren and CCBs, diuretics, ACEIs and ARBs exhibits synergistic anti-hypertensive effects. Many patients often require multidrug antihypertensive therapy. Aliskiren can play an important role as a RAAS-blocking drug in combination therapy.There is a need for longitudinal studies assessing aliskiren alone and in combination in treatment of persons with diabetes mellitus, metabolic syndrome and resistant hypertension to identify the specific categories of patients that would benefit more from direct renin blockade.

\section{References}

Allikmets, K. (2007). Aliskiren - an orally active renin inhibitor. Review of pharmacology, pharmacodynamics, kinetics, and clinical potential in the treatment of hypertension. Vasc Health Risk Manag., No. 3(6), 809-815. [in English].

Atlas, S. A. (2007). The renin-angiotensin aldosterone system: pathophysiological role and pharmacologic inhibition. J Manag Care Pharm, No. 13(8), 9-20. doi:10.18553/jmcp.2007.13.s8-b.9. [in English].

ESH/ESC. (2013) Guidelines for the management of arterial hypertension: The Task Force for the management of arterial hypertension of the European Society of Hypertension (ESH) and of the European Society of Cardiology (ESC). European Heart Journal, 34 (28), 2159 2219. doi.org/10.1093/eurheartj/eht151. [in English].

Ferrari, R. (2013). RAAS inhibition and mortality in hypertension. Glob Cardiol Science and Practice, No. 3, 269 - 278. doi: 10.5339/gcsp.2013.34. [in English].

Ghazi, L., Drawz, P. (2017). Advances in understanding the renin-angiotensin-aldosterone system (RAAS) in blood pressure control and recent pivotal trials of RAAS blockade in heart failure and diabetic nephropathy [version 1; referees: 3 approved], 6, 297. doi: 10.12688/f1000research.9692.1. [in English].

Katsi, V., Skalis, G., Pavlidis, A. N., Makris, T., Nihoyannopoulos, P., Tousoulis, D., Kallikazaros, I. (2015). Angiotensin receptor neprilysin inhibitor LCZ696: a novel targeted therapy for arterial hypertension? European Heart Journal - Cardiovascular Pharmacotherapy, No. 1 (4), 260-264. doi.org/10.1093/ehjcvp/pvv031. [in English].

Macit, C., Mercanoglu, G., Mercanoglu, F. (2015). A direct renin inhibitor aliskiren: reevaluation of effectiveness. J. Drug. Metab. Toxicol., No. 6, 185. doi: 10.4172/21577609.1000185. [in English]. 
Morishita, Y., Kusano, E. (2013). The effects of direct renin inhibitor, aliskiren, on arterial hypertension, chronic kidney disease and cardiovascular disease: optimal pharmacotherapy. Cardiovascular \& Hematological Agents in Medical Chemistry, No. 11(3), 77-82. [in English].

Müller, D. N., Luft, F. C. (2006). Direct Renin Inhibition with Aliskiren in Hypertension and Target Organ Damage. Clinical Journal of the American Society of Nephrology, No. 1, 221228. doi: 10.2215/CJN.01201005. [in English].

Musini, V. M., Lawrence, K. A. K., Fortin, P. M., Bassett, K., Wright, J. M. (2017). Blood pressure lowering efficacy of renin inhibitors for primary hypertension. Cochrane Database of Systematic Reviews, 4, CD007066. doi: 10.1002/14651858.CD007066.pub3. [in English].

Nissenson, A. R., Fine, R. N. (2017). Handbook of Dialysis Therapy (Fifth Edition), pages $i-$ ii. doi.org/10.1016/B978-0-323-39154-2.00099-0. [in English].

Pantzaris, N-D., Karanikolas, E., Tsiotsios, K., Velissaris, D. (2017). Renin Inhibition with Aliskiren: A Decade of Clinical Experience. J. Clin. Med., No. 6 (6), 61. doi:10.3390/jcm6060061. [in English].

Pool, J. L. (2007). Direct renin inhibition: Focus on aliskiren. J Manag Care Pharm, No. 13, 21-33. doi: 10.18553/jmcp.2007.13.s8-b.21. [in English].

Popov, V.V., Bulanov, N.A., Ivanov, G.G. (2012). Current target of antihypertensive therapy. data from clinical trials. part 1. Rational Pharmacotherapy in Cardiology, No. 8(1), 88-94. doi:10.20996/1819-6446-2012-8-1-88-94. [in English].

Sanoski, C.A. (2009). Aliskiren: an oral direct renin inhibitor for the treatment of hypertension. Pharmacotherapy, No. 29 (2), 193-212. doi: 10.1592/phco.29.2.193. [in English].

Saseen, J.J. (2013). Pharmacologic Management of Hypertension. Cardiovascular Therapeutics: A Companion to Braunwald's Heart Disease (Fourth Edition), 474-489. [in English].

Sen, S., Ufuktepe, B., Ozunal, Z. G., Uresin, Y. (2014). Renin inhibitors in diabetes and hypertension: an update. EXCLI Journal, No. 13, 1111-1119. [in English].

Sica D. A. (2009). Renin-Angiotensin Blockade: Therapeutic Agents. Textbook of NephroEndocrinology, 189-201. doi.org/10.1016/B978-0-12-373870-7.00015-6. [in English].

Staessen, J. A., Li, Y., Richart, T. (2006). Oral renin inhibitors. Lancet, 368, 1449 56. doi:10.1016/S0140-6736(06)69442-7. [in English].

Stanton, A., Jensen, C., Nussberger, J., O'Brien, E. (2003). Blood pressure lowering in essential hypertension with an oral renin inhibitor, aliskiren. Hypertension, No. 42 (6), 11371143. doi:10.1161/01.HYP.0000101688.17370.87. [in English].

Vaclavik, J., Sliva, J. (2014). ACE Inhibitors or Sartans in the Treatment of Hypertension: A Needless Discussion? J Hypertens, No. 3, 191. doi:10.4172/2167-1095.1000191. [in English]. Wal, P., Wal, A., Rai, A. K., Dixit, A. (2011). Aliskiren: An orally active renin inhibitor. J Pharm Bioallied Sci., No. 3 (2), 189-193. doi: 10.4103/0975-7406.80764. [in English].

Waldmeier, F., Glaenzel, U., Wirz, B., Oberer, L., Schmid, D., Seiberling, M., et al. (2007). Absorption, distribution, metabolism, and elimination of the direct renin inhibitor aliskiren in healthy volunteers. Drug Metab Dispos., No. 35, 1418-1428. doi:10.1124/dmd.106.013797. [in English].

Whelton, P.K., andt al. (2017). ACC/AHA/AAPA/ABC/ACPM/AGS/APhA/ASH/ASPC/ NMA/PCNA. Guideline for the Prevention, Detection, Evaluation, and Management of High Blood Pressure in Adults. A Report of the American College of Cardiology/American Heart 
Association Task Force on Clinical Practice Guidelines. Hypertension, 70 (6). doi.org/10.1161/HYP.0000000000000065. [in English].

World Health Organization. (2013). A global brief on hypertension 2013. Geneva, World Health Organization. [in English].

Zannad, F. (Moderator), Anker, S. D., Ruilope, L. M., Kosiborod, M. (Faculty). (2016). Optimising Renin-Angiotensin-Aldosterone System Inhibitor Therapy in Heart Failure and Resistant Hypertension: Challenges and Solutions. ESC 2016. Meeting summary. European Medical Journal, No.1 (4), 19-26. [in English]. 